\title{
Robust specification of the roughness penalty prior distribution in spatially adaptive Bayesian P-splines models
}

\author{
Astrid Jullion ${ }^{1}$ and Philippe Lambert ${ }^{1,2 *}$ \\ ${ }^{1}$ Institut de Statistique, Université catholique de Louvain, \\ Louvain-la-Neuve, Belgium \\ ${ }^{2}$ Unité d'épidémiologie, biostatistique et méthodes opérationnelles, \\ Faculté de Médecine, Université catholique de Louvain, Belgium.
}

July 12,2006

\begin{abstract}
The potential important role of the prior distribution of the roughness penalty parameter in the resulting smoothness of Bayesian P-splines models is considered. The recommended specification for that distribution yields models that can lack flexibility in specific circumstances. In such instances, these are shown to correspond to a frequentist P-splines model with a predefined and severe roughness penalty parameter, an obviously undesirable feature. It is shown that the specification of a hyperprior distribution for one parameter of that prior distribution provides the desired flexibility. Alternatively, a mixture prior can also be used. An extension of these two models by enabling adaptive penalties is provided. The posterior of all the proposed models can be quickly explored using the convenient Gibbs sampler.
\end{abstract}

${ }^{*}$ Correspondence to: Philippe Lambert, Université catholique de Louvain, Institut de Statistique, Voie du Roman Pays 20, B-1348 Louvain-la-Neuve (Belgium). E-mail: lambert@stat.ucl.ac.be Phone: +32-10-47.28.01 Fax: +32-10-47.30.32 


\section{Introduction}

Bayesian P-splines have recently become a widely used tool to describe the conditional mean of a response. Various authors have studied them either in normal (Ruppert et al., 2003; Berry et al., 2002; Lang and Brezger, 2004) or non-normal contexts (Fahrmeir et al., 2004; Lambert and Eilers, 2005; Lambert, 2005; Brezger and Lang, 2006). In the Bayesian P-splines model described in Lang and Brezger (2004), the prior distribution of the roughness penalty parameter $\tau_{\lambda}$ is taken to be a gamma with mean $a / b$ and variance $a / b^{2}$ with a small value for $b$. What we highlight in this paper is the influence that the choice of $b$ can have on the smoothness of the fitted curve. Indeed, we show that, in some specific circumstances, the results are highly sensitive to the value picked for $b$.

We propose two specifications that do not include the choice of such an influential hyperparameter. In the first specification, we treat the hyperparameters of the roughness penalty gamma conjugate prior as parameters to be estimated: this requires a reparametrisation beforehand. The second specification suggests to use as prior distribution for the penalty parameter a weighted sum of gamma distributions with different values for $b$. These two models make the fitting procedure automatic since it does not require to select a value for $b$. It is fast and easy to implement since one can simulate from the joint posterior using the Gibbs sampler.

We also propose an extension to adaptive penalties. This extension can be useful when the underlying function has a second derivative varying with $x$. In this case, adaptive penalties provide more flexibility and increase the quality of the fit. Such suggestions already exist in the literature (Denison et al., 2002; Lang and Brezger, 2004; Baladandayuthapani et al., 2005). Here, we propose to let the penalty parameter change at each knot by building them sequentially, as obtained by multiplying the previous one by a gamma random variable with mean 1 and a large variance. This construction yields a progressive evolution of the penalty parameter with $x$. With this specification, the Gibbs sampler can still be used. The presented techniques are illustrated by smoothing 
pharmacokinetics data.

The plan of our paper is as follows. In Section 2, we review the basic Bayesian P-splines model and we highlight the crucial role of the hyperparameters $a$ and $b$. In Section 3, we present our two alternative Bayesian P-splines model specifications. There, we comment the results of a simulation study comparing the performances of these two alternatives with the proposal made by Lang and Brezger (2004). Section 4 further extends our models to adaptive penalties. We conclude our presentation in Section 5 with a discussion.

\section{Basic Bayesian P-splines model}

Familiarity with P-splines is assumed. We refer to Eilers and Marx (1996) and to Ruppert et al. (2003) for further details. We give here a brief summary of the ideas provided in Eilers and Marx (1996).

A B-spline of degree $q$ consists of $q+1$ polynomial pieces, each of degree $q$. These polynomial pieces join at $q$ inner knots. The B-spline is positive on a domain spanned by $q+2$ knots and it is zero everywhere else. A property of B-splines is that the derivatives up to order $q-1$ are continuous at the joining points. Let $B_{j}(x ; q)$ denote the value at $x$ of the $j$ th B-spline of degree $q$ for a given equidistant grid of knots. The corresponding fitted curve $\hat{y}$ to data $\left\{\left(x_{i}, y_{i}\right)\right\}$ is a linear combination $\hat{y}(x)=\sum_{j=1}^{m} \hat{\theta}_{j} B_{j}(x ; q)$. Regression parameter estimates $\hat{\theta}_{j}$ can be obtained by minimising the least squares objective function

$$
S=\sum_{i=1}^{n}\left\{y_{i}-\sum_{j=1}^{m} \theta_{j} B_{j}\left(x_{i}\right)\right\}^{2} .
$$

Eilers and Marx (1996) suggest to take a large number of equidistant knots to ensure enough flexibility, and to counterbalance this by introducing a penalty on finite differences of adjacent B-splines coefficients, yielding the penalized objective function

$$
S=\sum_{i=1}^{n}\left\{y_{i}-\sum_{j=1}^{m} \theta_{j} B_{j}\left(x_{i}\right)\right\}^{2}+\lambda \sum_{j=k+1}^{m}\left(\Delta^{k} \theta_{j}\right)^{2},
$$

where $\Delta$ is the first-order difference operator. In terms of likelihood, the penalty 
appears as a term that we subtract from the $\log$-likelihood $l(y ; \theta)$ :

$$
l_{\text {pen }}=l(y ; \theta)-\frac{\lambda}{2} \sum_{j=k+1}^{m}\left(\Delta^{k} \theta_{j}\right)^{2} .
$$

\subsection{Model specification}

Let us remind the translation of the Eilers and Marx (1996) P-splines model in Bayesian terms (Lang and Brezger, 2004). The roughness penalty from the frequentist penalised likelihood approach translates into a prior distribution for the $r$ th order differences of successive B-splines parameters, $\theta_{j}$, yielding for a conditional normal response

$$
\begin{aligned}
\left(Y_{x} \mid \boldsymbol{\theta}, \tau\right) & \sim \mathcal{N}\left(\boldsymbol{b}_{x}^{\prime} \boldsymbol{\theta}, \tau^{-1}\right) \\
p(\tau) & \propto \tau^{-1} \\
p\left(\boldsymbol{\theta} \mid \tau_{\lambda}\right) & \propto \exp \left[-0.5 \tau_{\lambda} \boldsymbol{\theta}^{\prime} P \boldsymbol{\theta}\right] \\
\tau_{\lambda} & \sim \mathcal{G}(a, b)
\end{aligned}
$$

where

- $Y_{x}$ is a vector of responses, depending on $x$, having a conditional normal distribution with mean $\boldsymbol{b}_{x}^{\prime} \boldsymbol{\theta}$ and variance $\tau^{-1}$,

- $\boldsymbol{b}_{x}$ is the B-splines basis evaluated at $x$ and associated to a large number of equidistant knots,

- $\boldsymbol{\theta}$ is the vector of B-splines coefficients,

- $P=D^{\prime} D$ is the penalty matrix and $D$ the $r$ th-order difference matrix, yielding $\boldsymbol{\theta}^{\prime} P \boldsymbol{\theta}=\sum_{k}\left(\Delta^{r} \theta_{k}\right)^{2}$. Thus, for $r=2$, we have

$$
D=\left[\begin{array}{cccccc}
1 & -2 & 1 & 0 & \ldots & 0 \\
0 & 1 & -2 & 1 & \ldots & 0 \\
\vdots & & \ddots & \ddots & \ddots & \vdots \\
0 & 0 & \ldots & 1 & -2 & 1
\end{array}\right]
$$

- $\tau_{\lambda}$ is the roughness penalty parameter, 
- $\mathcal{G}(a, b)$ denotes a gamma distribution with mean $a / b$ and variance $a / b^{2}$. A large variance conjugate prior distribution is usually recommended and specified for $\tau_{\lambda}$, as suggested by Lang and Brezger (2004) by setting $a$ equal to 1 and $b$ equal to a small quantity $\left(10^{-5}\right.$, say).

\subsection{Conditional posterior distributions}

Given a set $\boldsymbol{y}=\left\{y_{x_{1}}, \ldots, y_{x_{n}}\right\}$ of independent observations, one can derive the conditional posterior distributions :

$$
\begin{aligned}
\left(\boldsymbol{\theta} \mid \tau, \tau_{\lambda} ; \boldsymbol{y}\right) & \sim \mathcal{N}\left(\tau \Sigma_{\theta} B^{\prime} R^{-1} \boldsymbol{y}, \Sigma_{\theta}\right) \\
(\tau \mid \mathrm{rest} ; \boldsymbol{y}) \equiv(\tau \mid \boldsymbol{\theta} ; \boldsymbol{y}) & \sim \mathcal{G}\left(0.5 n, 0.5(y-B \boldsymbol{\theta})^{\prime} R^{-1}(y-B \boldsymbol{\theta})\right) \\
\left(\tau_{\lambda} \mid \mathrm{rest} ; \boldsymbol{y}\right) \equiv\left(\tau_{\lambda} \mid \boldsymbol{\theta} ; \boldsymbol{y}\right) & \sim \mathcal{G}\left(a+0.5 \rho(P), b+0.5 \boldsymbol{\theta}^{\prime} P \boldsymbol{\theta}\right)
\end{aligned}
$$

where $\rho(P)$ is the rank of $P$,

$$
B=\left[\boldsymbol{b}_{x_{1}}, \ldots, \boldsymbol{b}_{x_{n}}\right]^{\prime}, \quad R=I_{n} \text { and } \Sigma_{\theta}^{-1}=\tau B^{\prime} R^{-1} B+\tau_{\lambda} P,
$$

and 'rest' generically denotes all the other parameters from the joint distribution. These formulas can be used with the Gibbs sampler to explore the joint posterior .

\subsection{Marginal posterior distributions}

The roughness penalty parameter $\tau_{\lambda}$ can be integrated out (Lambert, 2005), yielding the marginal posterior distribution

$$
p(\boldsymbol{\theta}, \tau \mid \boldsymbol{y}) \propto \frac{L(\boldsymbol{\theta}, \tau ; \boldsymbol{y}) p(\tau)}{\left(1+\frac{1}{2 b} \boldsymbol{\theta}^{\prime} P \boldsymbol{\theta}\right)^{a+0.5 \rho(P)}},
$$

where $L(\boldsymbol{\theta}, \tau ; \boldsymbol{y})$ is the likelihood. In a classical (frequentist) likelihood framework, this suggests working with a log-likelihood from which the following penalty is subtracted

$$
\operatorname{pen}(a, b) \doteq\left[a+\frac{1}{2} \rho(P)\right] \log \left(1+\frac{1}{2 b} \boldsymbol{\theta}^{\prime} P \boldsymbol{\theta}\right),
$$

where "” indicates equality up to an additive constant. This is to be compared with the classical penalised log-likelihood (Eilers and Marx, 1996)

$$
l_{\text {pen }}(\boldsymbol{\theta})=l(\boldsymbol{\theta})-\frac{1}{2} \lambda \theta^{\prime} P \boldsymbol{\theta},
$$


where $\lambda$ is usually selected using cross-validation (Wahba and Wold, 1975) or an information criterion such as the AIC. An alternative method, shown to be the best choice in some situations (Wecker and Ansley, 1983; Speckman and Sun, 2001), is the maximum marginal likelihood method. Whatever the chosen strategy, the selected $\lambda$ is a function of $\boldsymbol{y}$.

It is interesting to note the limiting behaviour of the conditional posterior distribution for $\boldsymbol{\theta}$ when the prior variance for $\tau_{\lambda}$ tends to infinity, as obtained by letting $b$ tend to $0^{+}$. In that case, the denominator in Equation (1) tends to infinity whatever the value of $a$, except if $\boldsymbol{\theta}^{\prime} P \boldsymbol{\theta}$ also tends to 0 . With a second order penalty, this happens when the fitted curve, $B \boldsymbol{\theta}$, tends to a line. Thus, what was first thought to be an expression of our ignorance concerning the appropriate penalty actually translates to an extremely severe roughness penalty.

Another interesting limiting behaviour of the penalty is

$$
\lim _{a, b \rightarrow+\infty} \operatorname{pen}(a, b) \doteq \frac{1}{2} \mu_{\tau_{\lambda}} \boldsymbol{\theta}^{\prime} P \boldsymbol{\theta} .
$$

It is associated to an informative gamma prior distribution for $\tau_{\lambda}$ with given mean $\mu_{\tau_{\lambda}}$ and a variance, $\mu_{\tau_{\lambda}} / b$, tending to zero. It corresponds to the classical penalised log-likelihood with penalty parameter equal to the prespecified prior mean $\mu_{\tau_{\lambda}}$.

These two extreme situations reveal the potential sensitivity of the results to the choice of $b$. This suggests that it should also be seen as a parameter in the model.

Another way to get the posterior distribution in Equation (1) is to consider the following equivalent model:

$$
\begin{aligned}
\left(Y_{x} \mid \boldsymbol{\theta}, \tau\right) & \sim \mathcal{N}\left(\boldsymbol{b}_{x}^{\prime} \boldsymbol{\theta}, \tau^{-1}\right) \\
p(\tau) & \propto \tau^{-1} \\
D \boldsymbol{\theta} & \sim t_{\nu=2 a}\left(\mathbf{0}, \frac{b}{a} I_{\rho(P)}\right)
\end{aligned}
$$

where $t_{\nu}(\boldsymbol{\mu}, \sigma)$ is the multivariate Student-t distribution with $\nu$ degrees of free- 
dom, mean $\boldsymbol{\mu}$ and variance-covariance matrix $\frac{\nu}{\nu-2} \Sigma$ when these two moments exist.

It highlights the crucial roles of $a$ and $b$. A small value for $2 a=\nu$ allows an occasionally very large second-order difference between successive components of $\boldsymbol{\theta}$, while the ratio $b / a=\delta$ determines the 'marginal' prior dispersion of these differences (with a variance equal to $b /(a-1)$ when it exists).

It suggests a reparametrisation of the basic P-splines model in Section 2.1 obtained by replacing $a$ and $b$ by $\nu / 2$ and $\delta \nu / 2$, respectively.

\subsection{Illustration}

An illustration of the limitations of the basic Bayesian P-splines model is obtained by applying it on 50 simulated data from the function $y_{x}=\mu_{x}+\epsilon_{x}$ with $\epsilon_{x} \sim \mathcal{N}(0,0.0169)$ and

$\mu_{x}=\left(1+\mathrm{e}^{-4(x-0.3)}\right)^{-1}+\left(1+\mathrm{e}^{3(x-0.2)}\right)^{-1}+\left(1+\mathrm{e}^{-4(x-0.7)}\right)^{-1}+\left(1+\mathrm{e}^{5(x-0.8)}\right)^{-1}$.

Different P-splines curves were fitted using cubic B-splines associated to 20 equally spaced knots on $(-2,2)$. It is suggested in Lang and Brezger (2004) to standardise the vector of responses $\boldsymbol{y}$ before estimation and to retransform the results afterwards. Figure 1-a- shows the fitted curves for different combinations (see legend) of $a$ and $b$. One can see the strong influence of the choice of the hyperparameter on the resulting fit. A too small value for $b$, initially thought to express our ignorance about the smoothing variance parameter $\tau_{\lambda}$, leads to an oversmoothed curve for the reasons explained in Section 2.3. A value for $b$ larger than suggested in the literature yields a satisfactory fit.

Note that when $a=b$, we still observe the same dependence of the results on the choice of the value picked for $a$ and $b$ (see Fig. 1-b-). Thus, some guidance should be provided to choose these hyperparameters (see Section 3). 

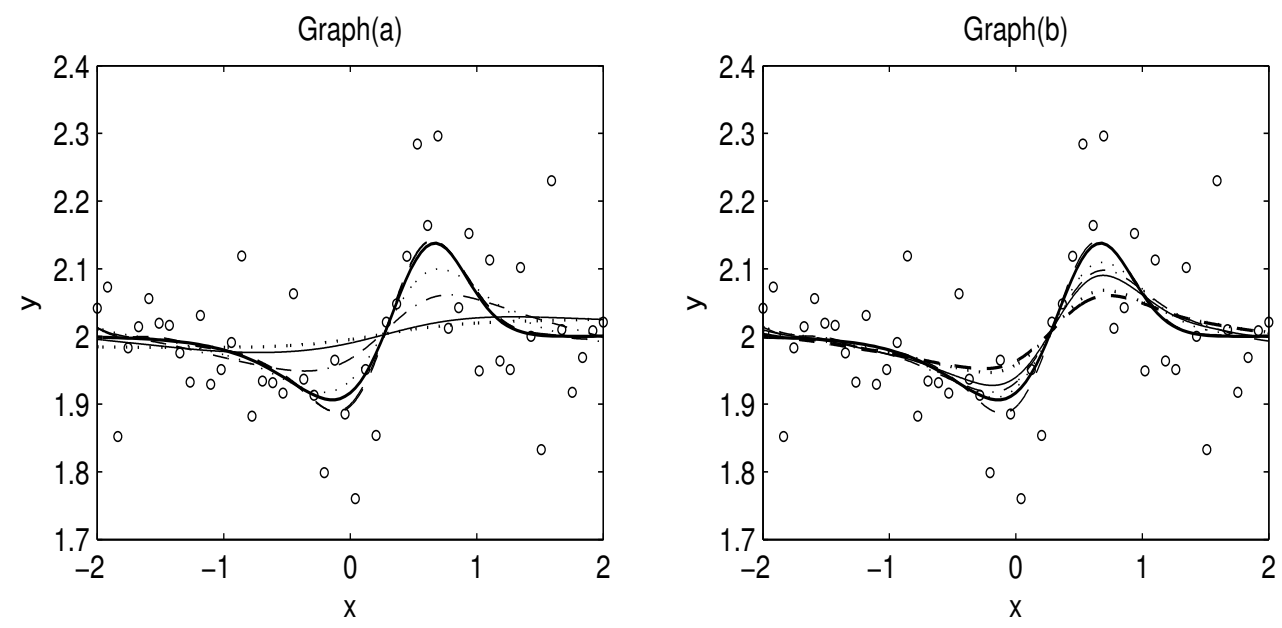

Figure 1: Illustrative data: fitted P-splines curves using the basic Bayesian P-splines model. Graph (a): $a=1$ combined with $b$ equal to 1 (dashed), 0.1 (thin dotted), 0.01 (dash-dotted) or 0.001 (thin solid). The fitted curves (thick dotted line) corresponding to $b$ equal to 0.0001 and 0.00001 cannot be graphically distinguished. Graph (b) : $a$ and $b$ both equal to 1 (dashed), 0.1 (thin dotted), 0.01 (dash-dotted), 0.001 (thin solid), 0.0001 (thick dotted) or 0.00001 (thick dashed). On both graphs, the underlying $\mu_{x}$ corresponds to the thick solid line. 


\section{Alternative specifications of the roughness penalty prior distribution}

\subsection{First specification: hyperpriors on the roughness prior}

As shown in the previous section, the choice of the parameters $a$ and $b$ for the prior distribution of the penalty parameter $\tau_{\lambda}$ has an important influence on the smoothness of the fitted curve.

Section 2.3 has highlighted the role of these quantities leading to a reparametrisation in terms of $\nu$ and $\delta$. These two parameters are difficult to prespecify and, hence, it is desirable to see them as parameters to be estimated. This is the topic of this section.

\subsubsection{Prior distribution on $\delta$}

A possible uninformative proper prior distribution for the dispersion parameter $\delta$ is

$$
\delta \sim \mathcal{G}\left(a_{\delta}, b_{\delta}\right)
$$

where we may take for instance $a_{\delta}=b_{\delta}$ equal to a small value. For a fixed value of $\nu$, we have the following conditional posterior distributions :

$$
\begin{aligned}
(\boldsymbol{\theta} \mid \text { rest } ; \boldsymbol{y}) \equiv\left(\boldsymbol{\theta} \mid \tau, \tau_{\lambda} ; \boldsymbol{y}\right) & \sim \mathcal{N}\left(\tau \Sigma_{\theta} B^{\prime} R^{-1} \boldsymbol{y}, \Sigma_{\theta}\right) \\
(\tau \mid \text { rest } ; \boldsymbol{y}) \equiv(\tau \mid \boldsymbol{\theta} ; \boldsymbol{y}) & \sim \mathcal{G}\left(0.5 n, 0.5(y-B \boldsymbol{\theta})^{\prime} R^{-1}(y-B \boldsymbol{\theta})\right) \\
\left(\tau_{\lambda} \mid \text { rest } ; \boldsymbol{y}\right) \equiv\left(\tau_{\lambda} \mid \boldsymbol{\theta}, \delta, \nu ; \boldsymbol{y}\right) & \sim \mathcal{G}\left(0.5 \nu+0.5 \rho(P), 0.5 \delta \nu+0.5 \boldsymbol{\theta}^{\prime} P \boldsymbol{\theta}\right) \\
(\delta \mid \text { rest } ; \boldsymbol{y}) \equiv\left(\delta \mid \tau_{\lambda}, \nu ; \boldsymbol{y}\right) & \sim \mathcal{G}\left(a_{\delta}+0.5 \nu, b_{\delta}+0.5 \nu \tau_{\lambda}\right) .
\end{aligned}
$$

These can be used directly with the Gibbs algorithm to sample from the joint posterior.

\subsubsection{Prior distribution on $\nu$}

We propose to take a uniform prior for $\nu$ on $(0, K)$ :

$$
p(\nu) \propto I_{(0, K)}(\nu),
$$


where $K>0$ is a large degrees of freedom yielding a Student-t density hardly distinguishable from the normal one.

Thus, the conditional posterior distribution for $\nu$ is

$$
p\left(\nu \mid \boldsymbol{\theta}, \tau, \tau_{\lambda}, \delta ; \boldsymbol{y}\right) \equiv p\left(\nu \mid \tau_{\lambda}, \delta ; \boldsymbol{y}\right) \propto \frac{1}{\Gamma\left(\frac{\nu}{2}\right)}\left(\delta \tau_{\lambda} \frac{\nu}{2}\right)^{\nu / 2} \mathrm{e}^{-\delta \tau_{\lambda} \frac{\nu}{2}} I_{(0, K)}(\nu) .
$$

In principle, any prior distribution can be considered for $\nu$. But none of them will provide an identifiable conditional posterior distribution for $\nu$. Therefore, a Metropolis-Hastings step will be required to generate a chain for $\nu$.

Alternatively, the Stirling's formula could be used in Equation (3) to approximate $\Gamma(\nu / 2)$ :

$$
\Gamma(\nu / 2)=\frac{2}{\nu} \Gamma(\nu / 2+1) \approx \sqrt{2 \pi}\left(\frac{\nu}{2}\right)^{\nu / 2-1 / 2} \mathrm{e}^{-\frac{\nu}{2}} .
$$

Substituting Equation (4) in Equation (3), one obtains

$$
\frac{1}{2 \sqrt{\pi}} \nu^{1 / 2} \mathrm{e}^{-\frac{1}{2}\left(\delta \tau_{\lambda}-\log \delta-\log \tau_{\lambda}-1\right) \nu} I_{(0, K)}(\nu) .
$$

As the Stirling's formula provides an excellent approximation ${ }^{1}$ to the gamma function for most of the relevant values of $\nu$, one can use the gamma density

$$
\mathcal{G}\left(1.5,0.5\left[\delta \tau_{\lambda}-\log \delta-\log \tau_{\lambda}-1\right]\right)
$$

(as suggested by Equation (5)) truncated to $(0, K)$ in an independence sampler to generate from the posterior distribution in Equation (3).

But, in our experience, no relevant information concerning the degrees of freedom can be obtained in practice, our MCMC simulations yielding a posterior distribution very close to a uniform on $(0, K)$. This is not surprising as, when $a_{\delta}=b_{\delta}$ are small,

$$
E\left(\delta \mid \boldsymbol{\theta}, \tau, \tau_{\lambda}, \delta, \nu ; \boldsymbol{y}\right)=\frac{a_{\delta}+0.5 \nu}{b_{\delta}+0.5 \nu \tau_{\lambda}} \approx \frac{1}{\tau_{\lambda}},
$$

suggesting that the second parameter of the truncated gamma approximating the conditional posterior distribution of $\nu$ is expected to take small values. This

\footnotetext{
${ }^{1}$ Stirling's formula underestimates the exact value of the gamma function by about $4(3,2)$ percents for an argument of the gamma function greater of equal to $3(4,5)$.
} 
corresponds to a posterior distribution with a large variance, as observed in our unreported examples.

Therefore, we simply suggest to fix $\nu$ to some value and to evaluate the sensitivity of the fitted curve to that choice.

\subsection{Second specification: a mixture prior for the penalty}

An alternative solution to avoid the sensitive choice of $b$ is to take as prior distribution for $\tau_{\lambda}$ a weighted sum of $M$ gamma distributions with different values for $b$.

This leads to the following model specification :

$$
\begin{aligned}
\left(Y_{x} \mid \boldsymbol{\theta}, \tau\right) & \sim \mathcal{N}\left(\boldsymbol{b}_{x}^{\prime} \boldsymbol{\theta}, \tau^{-1}\right) \\
p(\tau) & \propto \tau^{-1} \\
p\left(\boldsymbol{\theta} \mid \tau_{\lambda}\right) & \propto \exp \left[-0.5 \tau_{\lambda} \boldsymbol{\theta}^{\prime} \boldsymbol{P} \boldsymbol{\theta}\right] \\
\left(\tau_{\lambda} \mid \boldsymbol{p}\right) & \sim \sum_{m=1}^{M} p_{m} \mathcal{G}\left(a, b_{m}\right) \\
\boldsymbol{p} & \sim \mathcal{D}(\boldsymbol{u}),
\end{aligned}
$$

where $\left\{b_{1}, \ldots, b_{M}\right\}$ is a set of prespecified values, $\mathcal{D}$ stands for the Dirichlet distribution, and $\boldsymbol{u}^{\prime}=\left\{u_{1}, \ldots, u_{M}\right\}$ is a set of (small and equal) hyperprior parameters expressing our likely prior ignorance about the optimal choice for $b$.

The conditional posterior distributions are :

$$
\begin{aligned}
(\boldsymbol{\theta} \mid \text { rest } ; \boldsymbol{y}) \equiv\left(\boldsymbol{\theta} \mid \tau, \tau_{\lambda} ; \boldsymbol{y}\right) & \sim \mathcal{N}\left(\tau \Sigma_{\theta} B^{\prime} R^{-1} \boldsymbol{y}, \Sigma_{\theta}\right) \\
(\tau \mid \text { rest } ; \boldsymbol{y}) \equiv(\tau \mid \boldsymbol{\theta} ; \boldsymbol{y}) & \sim \mathcal{G}\left(0.5 n, 0.5(y-B \boldsymbol{\theta})^{\prime} R^{-1}(y-B \boldsymbol{\theta})\right) \\
\left(\tau_{\lambda} \mid \text { rest } ; \boldsymbol{y}\right) \equiv\left(\tau_{\lambda} \mid \boldsymbol{\theta}, \boldsymbol{p} ; \boldsymbol{y}\right) & \sim \sum_{m=1}^{M} p_{m} \mathcal{G}\left(a+0.5 \rho(P), b_{m}+0.5 \boldsymbol{\theta}^{\prime} \boldsymbol{P} \boldsymbol{\theta}\right) \\
(\boldsymbol{p} \mid \text { rest } ; \boldsymbol{y}) \equiv\left(\boldsymbol{p} \mid \tau_{\lambda} ; \boldsymbol{y}\right) & \propto \sum_{m=1}^{M} \frac{c_{m}}{\sum_{j=1}^{M} c_{j}} \mathcal{D}\left(u_{1}, \ldots, u_{m-1}, u_{m}+1, u_{m+1}, \ldots u_{M}\right),
\end{aligned}
$$

where

$$
c_{m}=\exp \left(-\tau_{\lambda} b_{m}\right) b_{m}^{a} \frac{\sum_{j=1}^{M} u_{j}}{u_{m}} .
$$




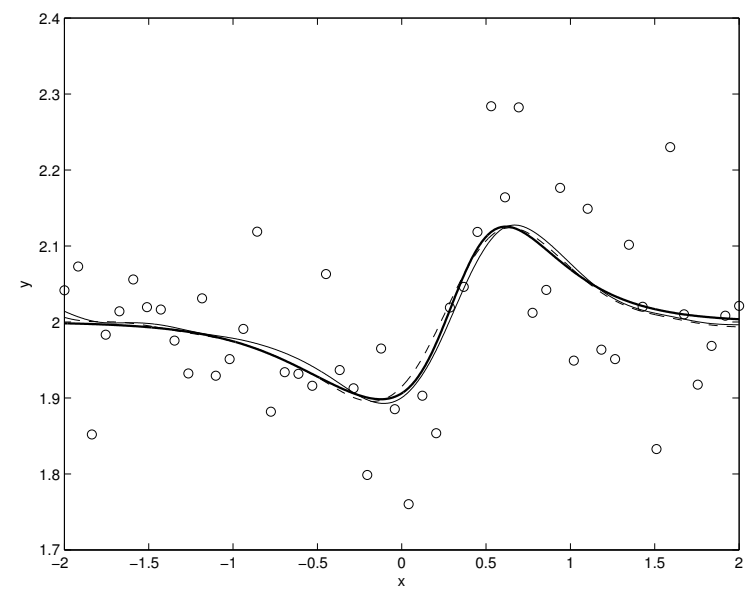

Figure 2: Illustrative data: fitted curves obtained using a Bayesian P-splines model combined with a mixture prior (dashed) or with a hyperprior on $\delta$ where $\nu=2 a=2$ and $a_{\delta}=b_{\delta}=0.0001$ (thin solid). The underlying $\mu_{x}$ corresponds to the thick solid line.

\subsection{Illustration}

Let us consider the same example as in Section 2.4. We use a Gibbs simulation with a chain of length 3,000 (and a burn-in of 1,000) to get the fitted curves shown in Fig. 2. For the mixture prior specification, we consider a grid of 33 values for $b$, logarithmically equally spaced between $10^{-6}$ and $10^{2}$. (A sensitivity analysis shows that the results do not depend on the choices made for $\nu, a_{\delta}$ or $b_{\delta}$, see Fig. 3.) We can see that the two fitted curves are close to each other and that both specifications provide a satisfactory fit.

Fig. 4-a- shows the distribution of $b=\delta \nu / 2$, as obtained from the Gibbs simulation in the first specification. The posterior distribution suggests pretty large values for $b$ (compared to the values recommended in the literature for that quantity). The mode of this distribution is 0.0031. Fig. 4-b- represents the posterior weight associated to each value of $b$ in the grid for the mixture prior specification: the posterior mode is 0.0032 .

The times required to run 1,000 iterations using (non-optimized) Matlab code on a Pentium IV $3.4 \mathrm{GHz}$ are $3.4 \mathrm{sec}$ for the basic Bayesian P-splines 

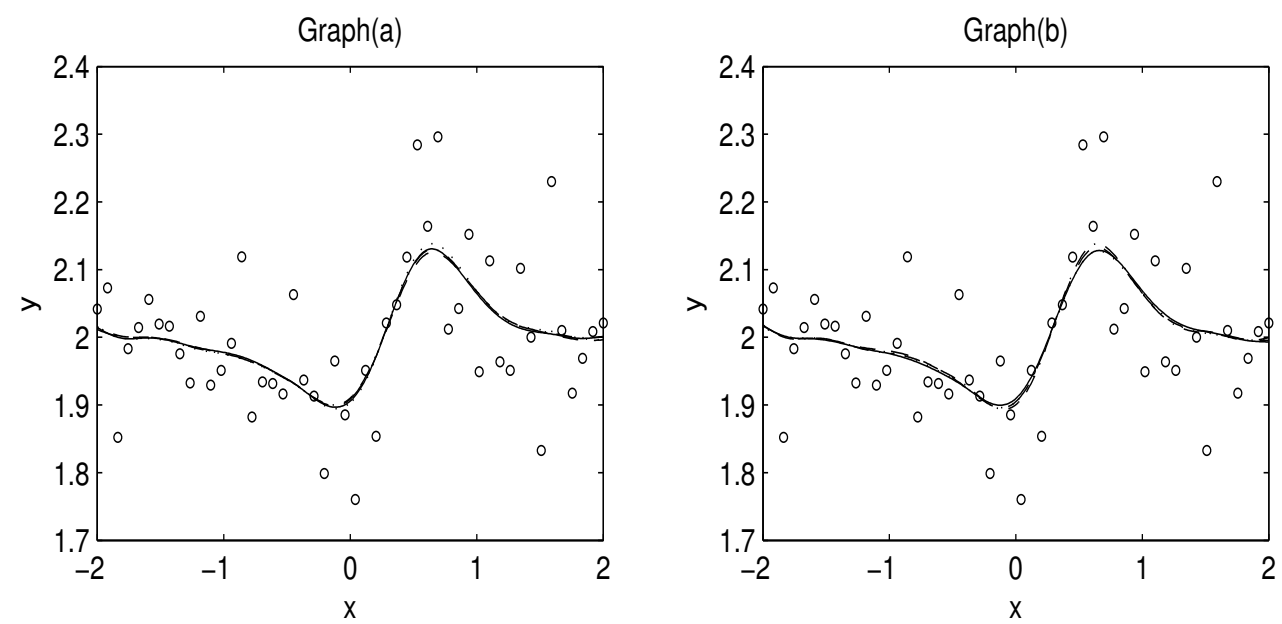

Figure 3: Illustrative data: sensitivity analysis of the fitted curve obtained with a Bayesian P-splines model combined with a hyperprior on $\delta$. Graph(a): sensitivity to the choice of $a_{\delta}=b_{\delta}: a_{\delta}=b_{\delta}=0.00001$ (solid line), $a_{\delta}=b_{\delta}=$ 0.0001 (dashed line), $a_{\delta}=b_{\delta}=0.001$ (dotted line), $a_{\delta}=b_{\delta}=0.01$ (dasheddotted line). Graph (b) : sensitivity to the choice of $\nu: \nu=2$ (solid line), $\nu=5$ (dashed line), $\nu=10$ (dotted line), $\nu=20$ (dashed-dotted line).
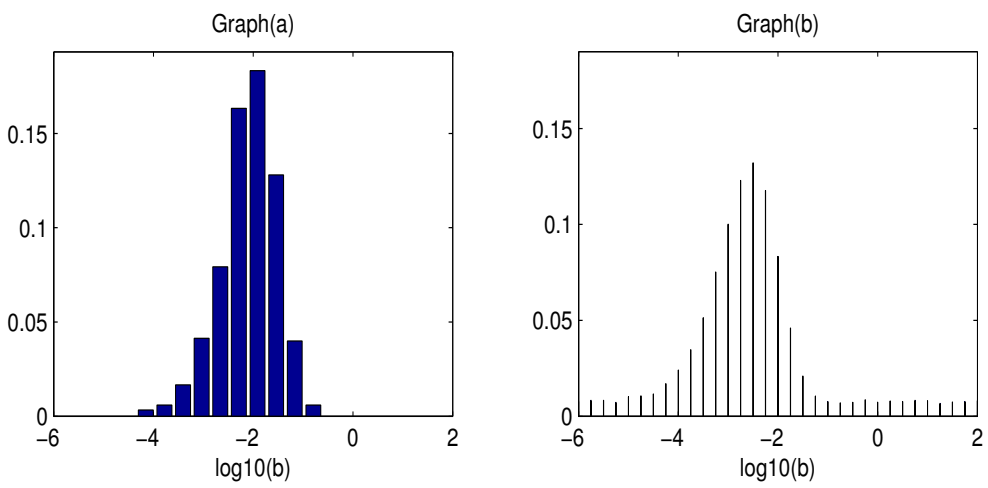

Figure 4: Illustrative data: posterior distribution of $b$ in the Bayesian P-splines model with (a) a hyperprior on $\delta$ where $b=\delta \nu / 2$; (b) a mixture prior. 
model ; $4.9 \mathrm{sec}$ for the model with a hyperprior on $\delta ; 4.9,7.2$ and $18.1 \mathrm{sec}$ with the mixture prior corresponding to, respectively, 5, 10 and 33 values for $b$. Thus, if computation time is an issue, the extension involving a hyperprior on $\delta$ is preferable.

\subsection{Simulation study}

We have performed a simulation study to compare the performances of the two proposed specifications for the penalty prior with the basic Bayesian P-splines model for different values of $b(0.1,0.01,0.001$ and 0.0001$)$. To simulate the data, we consider the same functions as in Lang and Brezger (2004), i.e. a linear function, $f(x)=\frac{1}{1.758} x$, a quadratic one, $f(x)=\frac{1}{2.75} x^{2}-1.5$, and a sinusoidal one $f(x)=\frac{1}{0.72} \sin (x)$. We also take the same values for the overall variance parameter $\sigma^{2}$, i.e. $\sigma=1,0.5$ and 0.33 . We simulated 100 repetitions for each of the nine combinations with $n=20$ design points $^{2}$ on an equidistant grid between -3 and 3 . We also considered the 'illustration function' presented in Section 2.4, i.e. $y_{x}=\mu_{x}+\epsilon_{x}$ with $\epsilon_{x} \sim \mathcal{N}(0,0.0169)$ and $\mu_{x}$ given by Equation (2) with $n=50$ design points between -2 and 2. In all cases, we took cubic B-splines with 20 equidistant knots.

For the first specification (cf. hyperprior on $\delta$ ), we take $a_{\delta}=b_{\delta}=0.0001$. For the mixture prior specification, a grid of 33 values logarithmically equally spaced between $10^{-6}$ and $10^{2}$ was taken for $b$.

The quality of the fit is measured by the logarithm of the empirical mean squared error given by

$$
M S E(\hat{f})=\frac{1}{20} \sum_{i=1}^{20}\left(f\left(x_{i}\right)-\hat{f}\left(x_{i}\right)\right)^{2},
$$

smaller values indicating better performances.

The results of the simulations are summarised in Figs. 5 to 8. Table 1 also provides the median of the posterior modes for $b$ under our two proposed priors.

In the linear case (see Fig. 5), the best results are obtained with the basic Bayesian P-splines model with the smallest value for $b(=0.0001)$. That small

\footnotetext{
${ }^{2} 100$ design points were considered in Lang and Brezger (2004)
} 


\begin{tabular}{cccc} 
Function & $\sigma$ & Hyperprior on $\delta$ & Mixture prior \\
\hline Linear & 1 & 0.049 & 0.010 \\
& 0.5 & 0.012 & 0.003 \\
& 0.33 & 0.005 & 0.003 \\
\hline Quadratic & 1 & 0.238 & 0.056 \\
& 0.5 & 0.125 & 0.032 \\
& 0.33 & 0.096 & 0.032 \\
\hline Sine & 1 & 0.170 & 0.056 \\
& 0.5 & 0.158 & 0.056 \\
& 0.33 & 0.140 & 0.056 \\
\hline Illustration & & 0.003 & 0.001
\end{tabular}

Table 1: Simulation study: median of the posterior modes for $b$ under our two proposed priors in the Bayesian P-splines model.
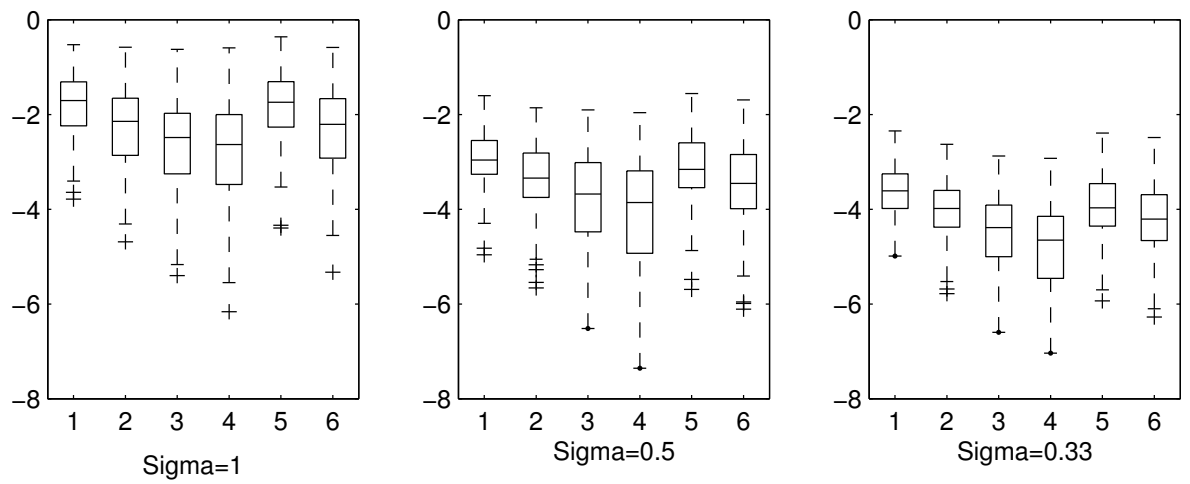

Figure 5: Simulation study: boxplots of $\log (M S E)$ for the linear function, $n=20$. The considered models are the basic Bayesian P-splines model with $b=0.1,0.01,0.001$ or 0.0001 (Priors 1 to 4 ) ; the Bayesian P-splines model with a hyperprior on $\delta$ (Prior 5) or a mixture prior (Prior 6). 

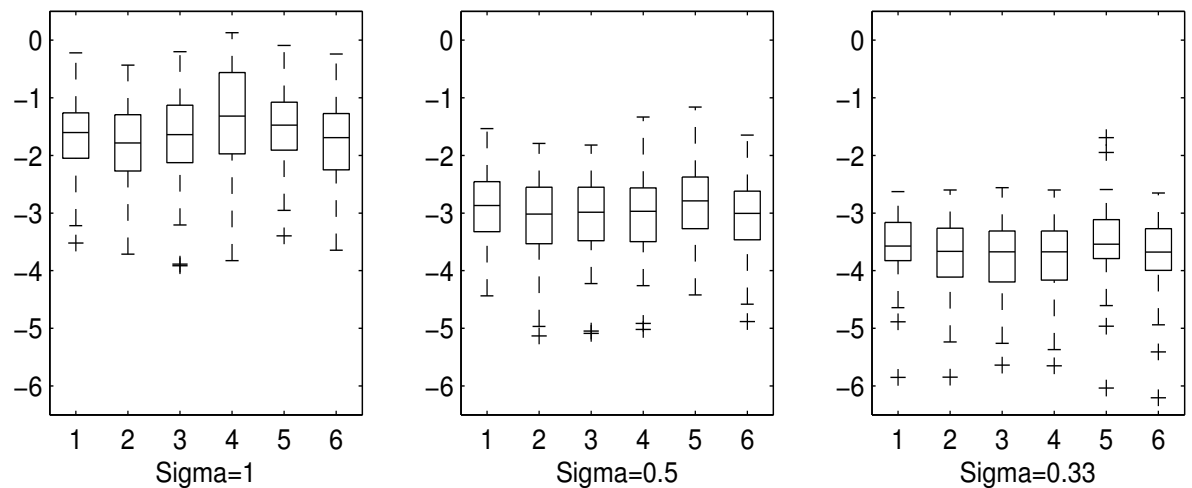

Figure 6: Simulation study: boxplots of $\log (M S E)$ for the quadratic function, $n=20$. The considered models are the basic Bayesian P-splines model with $b=0.1,0.01,0.001$ or 0.0001 (Priors 1 to 4) ; the Bayesian P-splines model with a hyperprior on $\delta$ (Prior 5) or a mixture prior (Prior 6).

value for $b$ implies a large penalty (cf. Section 2.3) with an expected linear fit at the limit when $b$ tend to $0^{+}$. Our two priors provide higher $\log (M S E)$ with better results for the mixture prior.

The influence of $b$ on the fit in the basic Bayesian P-splines model is negligible in the quadratic case, all specifications performing equally well (see Fig. $6)$.

It is not true anymore with the sine function (see Fig. 7) where the recommended small values for $b$ generate relatively large $M S E$ 's when the signal-tonoise ratio is low or very low. Then, larger values for $b$ should be considered to be competitive with our proposals for the prior. The same conclusions apply for the "illustration function" (see Fig. 8).

Dependence of the results on the sample size has been studied. Figs. 9 and 10 show the boxplots of $\log (M S E)$ for the sinus function with $n=50$ and $n=100$ design points. With $n=50$, all specifications perform equally well when $\sigma=0.33$ or $\sigma=0.5$. However, when $\sigma=1$, we still observe relatively large $M S E$ 's with the recommended small values for $b$. With larger sample size $(n=100)$, the choices for $a$ and $b$ no longer influence the fit.

Note that, for the first specification (hyperprior on $\delta$ ), a sensitivity analysis 

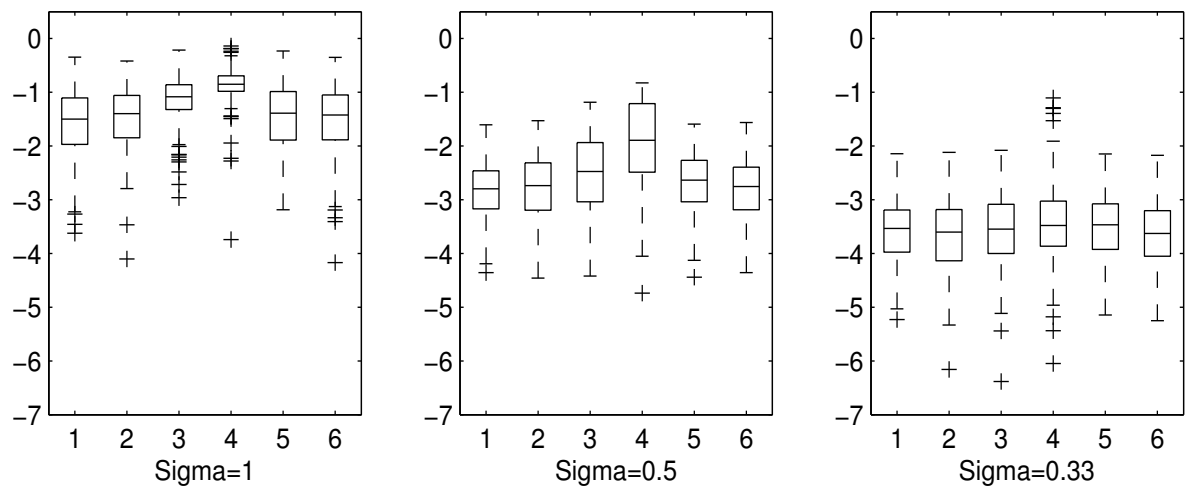

Figure 7: Simulation study: boxplots of $\log (M S E)$ for the sine function, $n=20$. The considered models are the basic Bayesian P-splines model with $b=0.1,0.01$, 0.001 or 0.0001 (Priors 1 to 4 ) ; the Bayesian P-splines model with a hyperprior on $\delta$ (Prior 5) or a mixture prior (Prior 6).

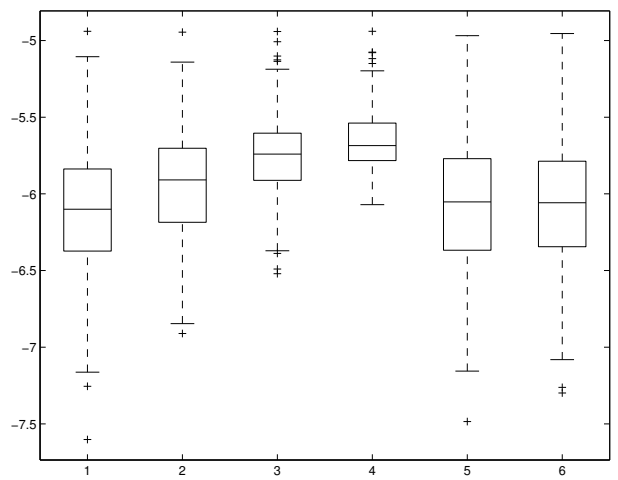

Figure 8: Simulation study: boxplots of $\log (M S E)$ for the illustration function, $n=50$. The considered models are the basic Bayesian P-splines model with $b=0.1,0.01,0.001$ or 0.0001 (Priors 1 to 4 ) ; the Bayesian P-splines model with a hyperprior on $\delta$ (Prior 5) or a mixture prior (Prior 6). 

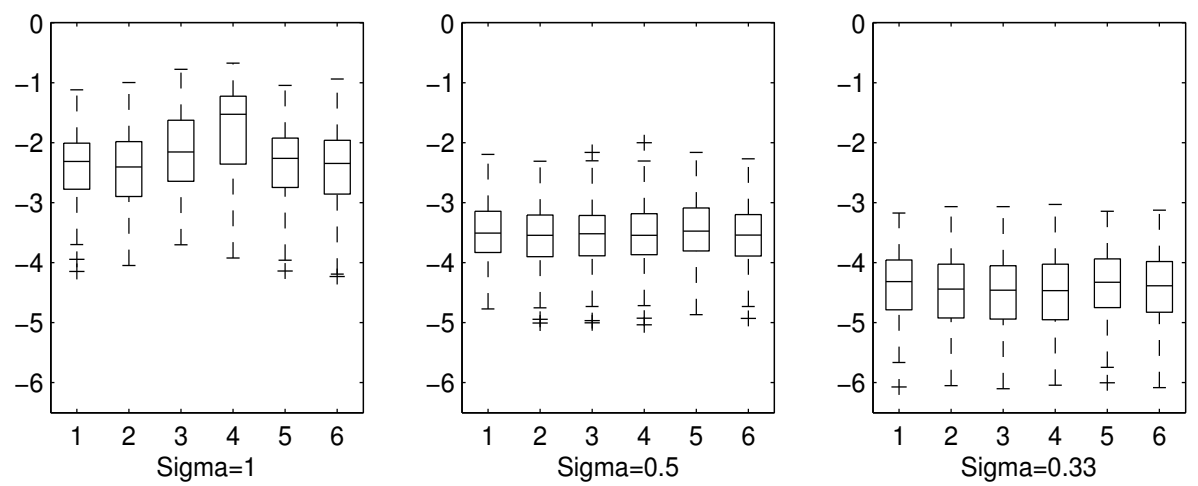

Figure 9: Simulation study: boxplots of $\log (M S E)$ for the sine function, $n=50$. The considered models are the basic Bayesian P-splines model with $b=0.1,0.01$, 0.001 or 0.0001 (Priors 1 to 4) ; the Bayesian P-splines model with a hyperprior on $\delta$ (Prior 5) or a mixture prior (Prior 6).

revealed no significant influence of $a_{\delta}=b_{\delta}$ on the fit as can be seen in Fig. 11, where boxplots of the $\log (M S E)$ are drawn for the sinus function with different values for $a_{\delta}$ and $b_{\delta}(0.01,0.001,0.0001$ and 0.00001$)$. The robustness of the fit to the choice of $a_{\delta}$ and $b_{\delta}$ for the illustration function can be assessed from Fig. 3.

This simulation study suggests that the recommendation to take a very small value for $b$ may reveal not to be a good choice in specific circumstances (such as a small sample size and/or data with a poor signal-to-noise ratio). This would, of course, be revealed by a sensitivity analysis of the results to the choice of $b$. However, the ease with which our alternative specifications can be implemented makes them attractive while relieving us from such an analysis.

\section{$4 \quad$ P-splines model with adaptive penalties}

An adaptive penalty is desirable to smooth function with a second derivative significantly varying with $x$. 

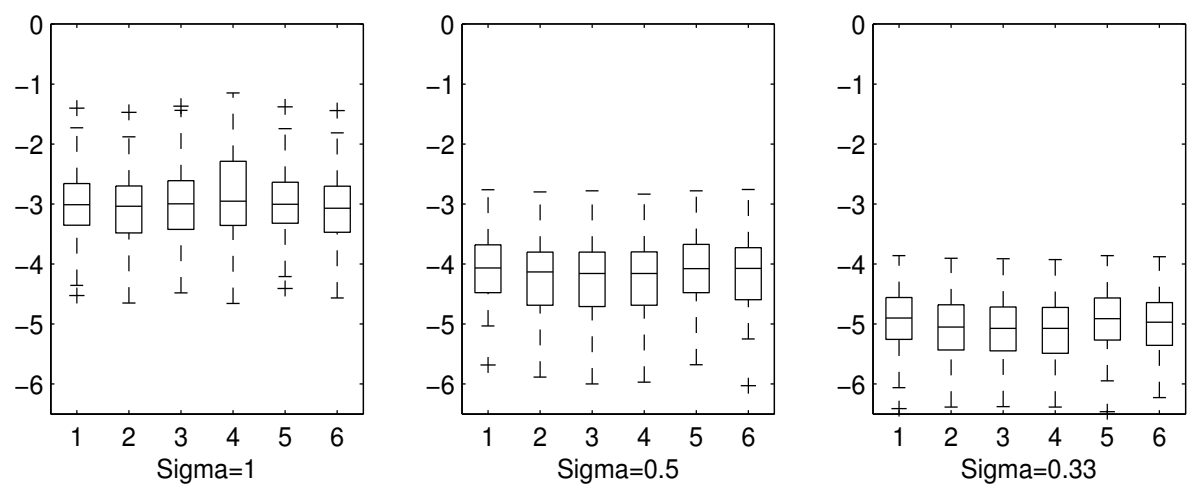

Figure 10: Simulation study: boxplots of $\log (M S E)$ for the sine function, $n=$ 100. The considered models are the basic Bayesian P-splines model with $b=0.1$, 0.01, 0.001 or 0.0001 (Priors 1 to 4 ) ; the Bayesian P-splines model with a hyperprior on $\delta$ (Prior 5) or a mixture prior (Prior 6).
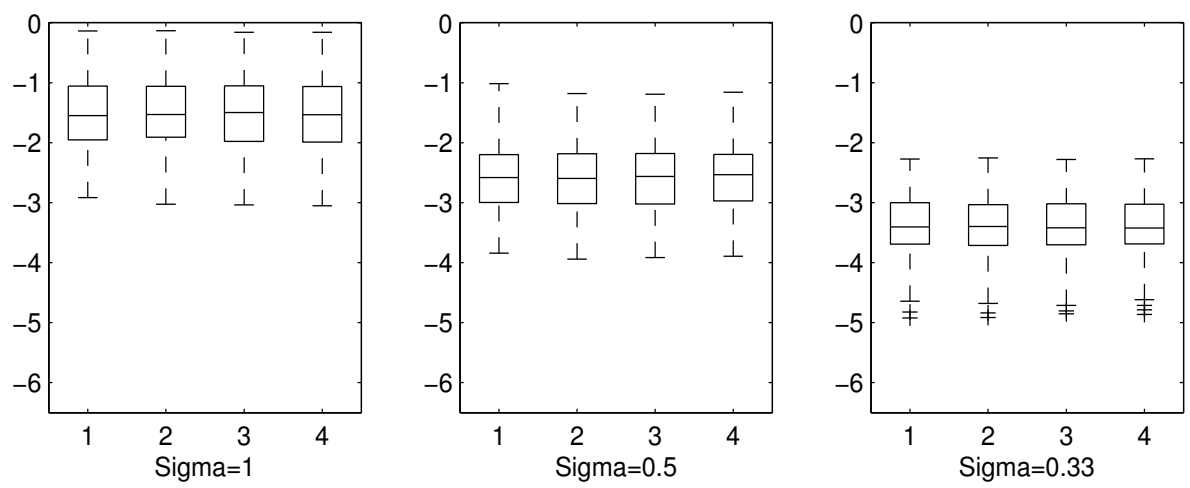

Figure 11: Simulation study: boxplots of $\log (M S E)$ for the sine function, $n=$ 20. The considered model is the Bayesian P-splines model with a hyperprior on $\delta$ with (1) $a_{\delta}=b_{\delta}=0.01,(2) a_{\delta}=b_{\delta}=0.001,(3) a_{\delta}=b_{\delta}=0.0001$, (4) $a_{\delta}=b_{\delta}=0.00001$. 


\subsection{Adaptive penalties combined with a hyperprior for $\delta$}

A progressive evolution of the penalty parameter with $x$ can be obtained with the following model specification:

$$
\begin{aligned}
\left(Y_{x} \mid \boldsymbol{\theta}, \tau\right) & \sim \mathcal{N}\left(\boldsymbol{b}_{x}^{\prime} \boldsymbol{\theta}, \tau^{-1}\right) \\
p(\tau) & \propto \tau^{-1} \\
p\left(\boldsymbol{\theta} \mid \tau_{\lambda}, \Lambda\right) & \propto \exp \left[-0.5 \tau_{\lambda} \sum_{k=r+1}^{K}\left(\prod_{l=r+1}^{k} \lambda_{l}\right)\left(\Delta^{r} \theta_{k}\right)^{2}\right] \\
& =\exp \left[-0.5 \tau_{\lambda} \sum_{k=r+1}^{K} \lambda^{(k)}\left(\Delta^{r} \theta_{k}\right)^{2}\right] \\
& =\exp \left[-0.5 \tau_{\lambda} \boldsymbol{\theta}^{\prime} D^{\prime} \Lambda D \boldsymbol{\theta}\right] \\
\lambda_{k} & \sim \mathcal{G}(\omega, \omega) \quad \text { when } k>r+1 ; \lambda_{r+1}=1 \\
\left(\tau_{\lambda} \mid \delta\right) & \sim \mathcal{G}(0.5 \nu, 0.5 \delta \nu) \\
\delta & \sim \mathcal{G}\left(a_{\delta}, b_{\delta}\right),
\end{aligned}
$$

where

$$
\Lambda=\operatorname{diag}\left(\lambda^{(r+1)}, \ldots, \lambda^{(K)}\right) .
$$

That diagonal matrix contains a penalty parameter for each $r$ th-order difference between successive components of $\boldsymbol{\theta}$. They are obtained sequentially by multiplying the previous one by a gamma random variable with mean 1 and (an arbitrarily large) variance $\omega^{-1}$. That construction yields a progressive evolution of the penalty parameter with $x$.

Note that this proposal differs from Lang and Brezger (2004) where no 'smoothness' is imposed on the roughness penalty coefficient. Such a smoothness was imposed in Baladandayuthapani et al. (2005) on the log-scale of the variance. Unfortunately, it required the use of the Metropolis-Hastings as some conditional posterior distributions cannot be identified. Here, the Gibbs sampler can be used as all the conditional distributions can be identified:

$$
\begin{aligned}
\left(\boldsymbol{\theta} \mid \tau, \tau_{\lambda}, \delta, \boldsymbol{\lambda} ; \boldsymbol{y}\right) & \sim \mathcal{N}\left(\tau \Sigma_{\theta} B^{\prime} R^{-1} \boldsymbol{y}, \Sigma_{\theta}\right) \\
(\tau \mid \text { rest } ; \boldsymbol{y}) \equiv(\tau \mid \boldsymbol{\theta} ; \boldsymbol{y}) & \sim \mathcal{G}\left(0.5 n, 0.5(y-B \boldsymbol{\theta})^{\prime} R^{-1}(y-B \boldsymbol{\theta})\right) \\
\left(\lambda_{l} \mid \text { rest } ; \boldsymbol{y}\right) \equiv\left(\lambda_{l} \mid \boldsymbol{\theta}, \tau_{\lambda}, \boldsymbol{\lambda}_{-l} ; \boldsymbol{y}\right) & \stackrel{l>r+1}{\sim} \mathcal{G}\left(\omega+\frac{K-l+1}{2}, \omega+\frac{\tau_{\lambda}}{2} \sum_{k=l}^{K} \frac{\lambda^{(k)}}{\lambda_{l}}\left(\Delta^{r} \theta_{k}\right)^{2}\right)
\end{aligned}
$$




$$
\begin{aligned}
\left(\tau_{\lambda} \mid \mathrm{rest} ; \boldsymbol{y}\right) \equiv\left(\tau_{\lambda} \mid \boldsymbol{\theta}, \delta, \boldsymbol{\lambda} ; \boldsymbol{y}\right) & \sim \mathcal{G}\left(0.5 \nu+0.5 \rho(P), 0.5 \delta \nu+0.5 \boldsymbol{\theta}^{\prime} D^{\prime} \Lambda D \boldsymbol{\theta}\right) \\
\left(\delta \mid \tau, \tau_{\lambda}, \boldsymbol{\lambda} ; \boldsymbol{y}\right) & \sim \mathcal{G}\left(a_{\delta}+0.5 \nu, b_{\delta}+0.5 \nu \tau_{\lambda}\right)
\end{aligned}
$$

where

$$
\Sigma_{\theta}^{-1}=\tau B^{\prime} R^{-1} B+\tau_{\lambda} D^{\prime} \Lambda D
$$

\subsection{Adaptive penalties with a mixture prior for the reference penalty}

If, instead, a mixture prior for the reference penalty is considered (cf. Section 3.2 ), we get the following model specification:

$$
\begin{aligned}
\left(Y_{x} \mid \boldsymbol{\theta}, \tau\right) & \sim \mathcal{N}\left(\boldsymbol{b}_{x}^{\prime} \boldsymbol{\theta}, \tau^{-1}\right) \\
p(\tau) & \propto \tau^{-1} \\
p\left(\boldsymbol{\theta} \mid \tau_{\lambda}, \Lambda\right) & \propto \exp \left[-0.5 \tau_{\lambda} \boldsymbol{\theta}^{\prime} D^{\prime} \Lambda D \boldsymbol{\theta}\right] \\
\lambda_{k} & \sim \mathcal{G}(\omega, \omega) \text { when } k>r+1 ; \lambda_{r+1}=1 \\
\left(\tau_{\lambda} \mid \boldsymbol{p}\right) & \sim \sum_{m=1}^{M} p_{m} \mathcal{G}\left(a, b_{m}\right) \\
\boldsymbol{p} & \sim \mathcal{D}(\boldsymbol{u}) .
\end{aligned}
$$

The conditional posterior distributions are:

$$
\begin{aligned}
(\boldsymbol{\theta} \mid \text { rest } ; \boldsymbol{y}) \equiv\left(\boldsymbol{\theta} \mid \tau, \tau_{\lambda} \boldsymbol{\lambda} ; \boldsymbol{y}\right) & \sim \mathcal{N}\left(\tau \Sigma_{\theta} B^{\prime} R^{-1} \boldsymbol{y}, \Sigma_{\theta}\right) \\
(\tau \mid \text { rest } ; \boldsymbol{y}) \equiv(\tau \mid \boldsymbol{\theta} ; \boldsymbol{y}) & \sim \mathcal{G}\left(0.5 n, 0.5(y-B \boldsymbol{\theta})^{\prime} R^{-1}(y-B \boldsymbol{\theta})\right) \\
\left(\lambda_{l} \mid \text { rest } ; \boldsymbol{y}\right) \equiv\left(\lambda_{l} \mid \boldsymbol{\theta}, \tau_{\lambda}, \boldsymbol{\lambda}_{-l} ; \boldsymbol{y}\right) & \stackrel{l>r+1}{\sim} \mathcal{G}\left(\omega+\frac{K-l+1}{2}, \omega+\frac{\tau_{\lambda}}{2} \sum_{k=l}^{K} \frac{\lambda^{(k)}}{\lambda_{l}}\left(\Delta^{r} \theta_{k}\right)^{2}\right) \\
\left(\tau_{\lambda} \mid \text { rest } ; \boldsymbol{y}\right) \equiv\left(\tau_{\lambda} \mid \boldsymbol{\theta}, \boldsymbol{\lambda}, \boldsymbol{p} ; \boldsymbol{y}\right) & \sim \sum_{m=1}^{M} p_{m} \mathcal{G}\left(a+0.5 \rho(P), b_{m}+0.5 \boldsymbol{\theta}^{\prime} D^{\prime} \Lambda D \boldsymbol{\theta}\right) \\
(\boldsymbol{p} \mid \text { rest } ; \boldsymbol{y}) \equiv\left(\boldsymbol{p} \mid \tau_{\lambda} ; \boldsymbol{y}\right) & \propto \sum_{m=1}^{M} \frac{c_{m}}{\sum_{j=1}^{M} c_{j}} \mathcal{D}\left(u_{1}, \ldots, u_{m}+1, \ldots u_{M}\right),
\end{aligned}
$$

where

$$
c_{m}=\exp \left(-\tau_{\lambda} b_{m}\right) b_{m}^{a} \frac{\sum_{j=1}^{M} u_{j}}{u_{m}} .
$$




\begin{tabular}{cccccc}
$\mathrm{x}$ & $\mathrm{y}$ & $\mathrm{x}$ & $\mathrm{y}$ & $\mathrm{x}$ & $\mathrm{y}$ \\
\hline 0 & 0 & $4.47 \mathrm{e}-02$ & 3.142 & $2.89 \mathrm{e}-01$ & 3.278 \\
$2.74 \mathrm{e}-03$ & 0.399 & $5.98 \mathrm{e}-02$ & 3.742 & $3.72 \mathrm{e}-01$ & 2.628 \\
$6.25 \mathrm{e}-03$ & 1.138 & $7.90 \mathrm{e}-02$ & 3.519 & $4.77 \mathrm{e}-01$ & 2.292 \\
$1.07 \mathrm{e}-02$ & 1.511 & $1.03 \mathrm{e}-01$ & 3.067 & $6.11 \mathrm{e}-01$ & 2.359 \\
$1.64 \mathrm{e}-02$ & 2.005 & $1.34 \mathrm{e}-01$ & 3.870 & $7.82 \mathrm{e}-01$ & 2.011 \\
$2.36 \mathrm{e}-02$ & 2.957 & $1.74 \mathrm{e}-01$ & 2.977 & $1.00 \mathrm{e}+00$ & 1.717 \\
$3.29 \mathrm{e}-02$ & 3.421 & $2.25 \mathrm{e}-01$ & 3.093 & &
\end{tabular}

Table 2: Simulated pharmacokinetics data corresponding to a two-compartment model with multiplicative log-normal error: $y_{x}=\mu_{x} \exp \left(\epsilon_{x}\right)$ with $\epsilon_{x} \sim$ $\mathcal{N}(0,0.01)$ and $\mu_{x}=\frac{A k_{a}}{k_{a}-k_{e}}\left[\exp \left(-k_{e} x\right)-\exp \left(-k_{a} x\right)\right]$, where $A=3.74, k_{e}=$ $0.78, k_{a}=50$.

\subsection{Illustration}

A challenging illustration of the model performances is obtained by applying it on pharmacokinetics data giving the measured evolution of the concentration of a drug in the plasma over time (see Table 2). The measurement times are approximately equally spaced on the log-scale. Most measurements are taken at early times where the underlying curvature has the largest gradient.

Fig. 12-a- shows the fitted curves obtained with the first specification (thin solid line, cf. Section 3.1) and with the mixture prior one (dashed line, cf. Section 3.2) but without adaptive penalties. The underlying $\mu_{x}$ corresponds to the thick solid line. These curves were obtained with a chain of length 3,000 (and a burn-in of 1,000) generated using the Gibbs sampler. For the first specification, we use $\nu=2$ and $a_{\delta}=b_{\delta}=0.0001$. For the mixture prior, we consider a grid of 33 values for $b$, logarithmically equally spaced between $10^{-6}$ and $10^{2}$. In both cases, we obtain a wiggly curve that captures part of the early quick rise in the response, but with overfitting at later times. It is obviously a compromise between ideally a large value for $b$ for small times (where the curvature is large) and a small value for $b$ for later times (where the target 

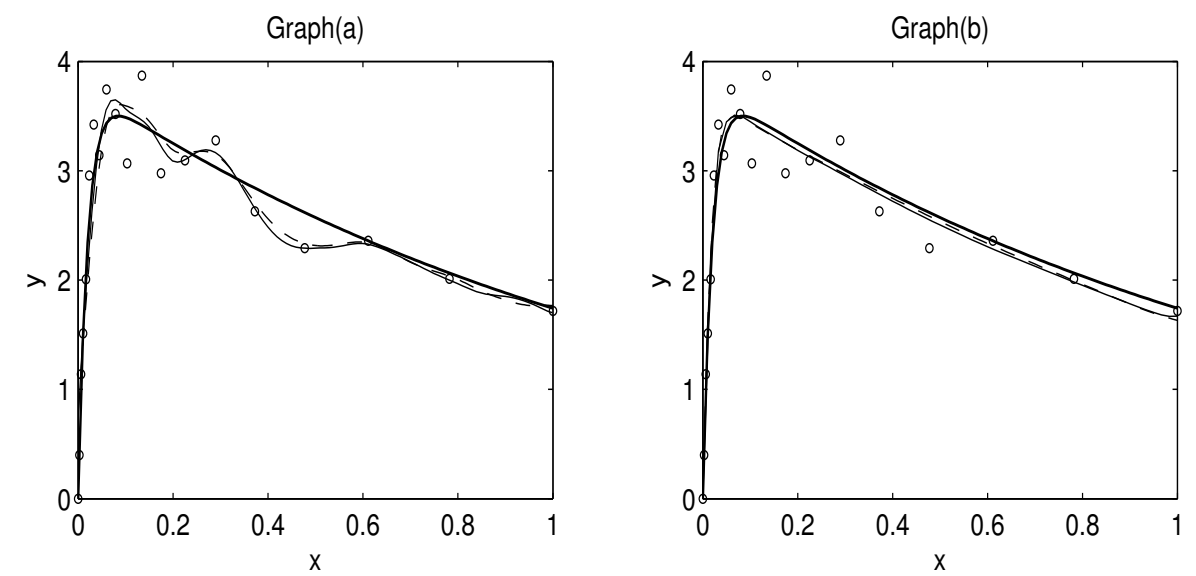

Figure 12: Pharmacokinetics data from Table 2: fitted curves using the Bayesian P-splines models with a hyperprior on $\delta$ (thin solid line) or a mixture prior (dashed line) in combination with (a) a non-adaptive penalty (b) adaptive penalties. The underlying $\mu_{x}$ corresponds to the thick solid line.

curve is approximately a line).

Fig. 12-b- shows the fitted curves obtained with adaptive penalties combined with the hyperprior on $\delta$ (thin solid line, cf. Section 4.1) and with the mixture prior (dashed line, cf. Section 4.2). The two fitted curves are hardly distinguishable from the target curve in the rising phase that requires flexibility and, thus, a small penalty. A linear pattern is obtained for later times as a consequence of large penalty parameters.

The posterior distribution of $\log (b=\delta \nu / 2)$ under each of the two prior specifications with adaptive penalties are given in Fig. 13. The posterior modes under the hyperprior for $\delta$ and the mixture prior specifications are equal to 2.85 (see Fig 13-a-) and 2.25 (see Fig 13-b-), respectively. Note that a larger grid (33 values for $b$, logarithmically equally spaced between $10^{-6}$ and $10^{5}$ ) than before (with an upper limit set previously at $10^{2}$ ) had to be considered for $b$.

The times required to run 1,000 iterations using (non-optimized) Matlab code on a Pentium IV $3.4 \mathrm{GHz}$ are 1.8 and $3.8 \mathrm{sec}$ with the the model with a hyperprior on $\delta$ with, respectively, a non-adaptive penalty and adaptive penalties ; 16.7 and $19.1 \mathrm{sec}$ for the model with a mixture prior corresponding to 33 

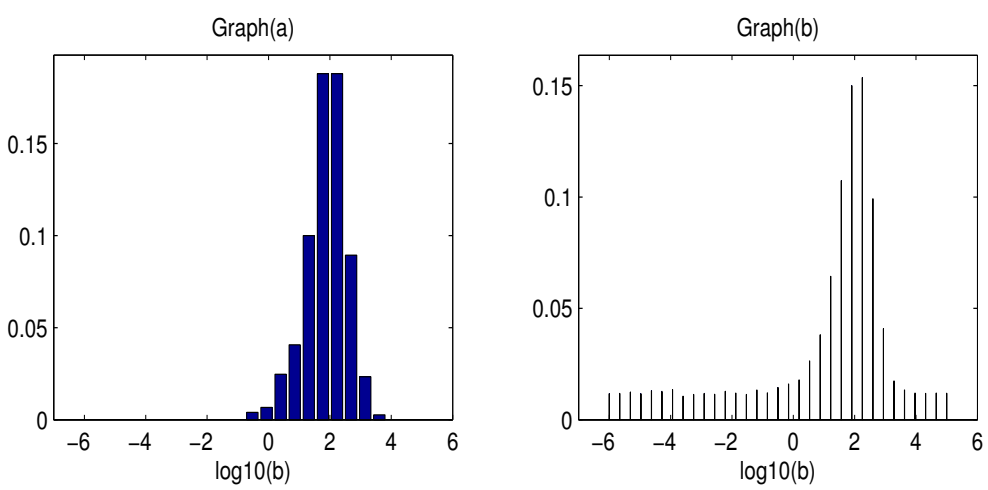

Figure 13: Simulated pharmacokinetics data from Table 2: posterior distribution of $\log (b=\delta \nu / 2)$ under the hyperprior (for $\delta$ ) and the mixture prior specifications with adaptive penalties.

values for $b$ with, respectively, a non-adaptive penalty and adaptive penalties. Again, if computation time is an issue, the extension involving a hyperprior on $\delta$ is preferable.

Note that, in some situations, applying an adequate transformation to the data may avoid the need to use the two extensions proposed in this paper. For instance, if we apply the logarithm to these illustration data (both on $x$ and $y$ ), we can get a satisfactory fit with a basic P-splines model (results not shown). Applying some transformations is then an option that should be considered when fitting data.

\subsection{Simulation study}

We have performed a simulation study to compare the performances of the models with adaptive penalties. To simulate the data, we consider the function: $y_{x}=\mu_{x} \exp \left(\epsilon_{x}\right)$ with $\epsilon_{x} \sim \mathcal{N}(0,0.01)$ and $\mu_{x}=\frac{A k_{a}}{k_{a}-k_{e}}\left[\exp \left(-k_{e} x\right)-\exp \left(-k_{a} x\right)\right]$, where $A=3.74, k_{e}=0.78, k_{a}=50$. We simulate 100 repetitions with $n=20$ design points in $(0,1)$, approximately equidistant on the log-scale. We consider cubic B-splines with 20 equidistant knots.

Fig. 14 gives the boxplot of $\log (M S E)$ for different models (see legend). Bayesian models with robust prior specification provide smaller $M S E$ than the 


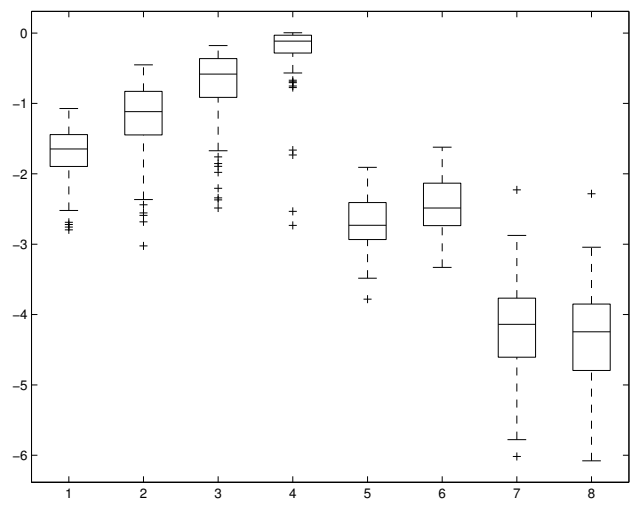

Figure 14: Simulation study: boxplots of $\log (M S E)$ for the pharmacokinetics function. Model 1 to 4 are the basic Bayesian P-splines model with $a=b=0.1$, $a=b=0.01, a=b=0.001$ and $a=b=0.0001 ;$ the Bayesian P-splines model with a mixture prior (Model 5) or a hyperprior on $\delta$ (Model 6); the Bayesian P-splines model with adaptive penalties combined with a mixture prior (Model 7) or a hyperprior on $\delta$ (Model 8).

basic Bayesian models whatever the choice made for $a$ and $b$. The Bayesian model with a mixture prior yields $M S E$ 's that are a bit smaller than the ones obtained with the hyperprior on $\delta$ when the penalty is non-adaptive.

Adaptive penalties considerably improve the fit without significant differences between Models 7 and 8 .

\section{Application on real data}

We apply the previous models to a real data set coming from a Positron Emission Tomography (PET) study. The radioactivity level is measured during the time-length of the scan in different parts of the brain. Based on these time-activity curves, some important clinical measures are derived, such as the binding potential or the receptor occupancy (van Warde, 2000). Due to noise, it is often necessary to smooth the time-course of radioactivity measured in PET scans prior to any quantitative analysis. On Fig. 15, Graph(a) shows the fit of different models (see legend) to a time-activity curve. One can see that adap- 

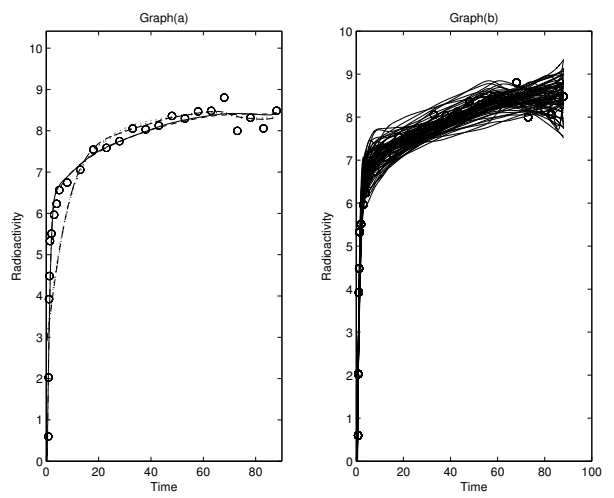

Figure 15: Time-activity data. Graph(a) : fitted curves using the Bayesian P-splines models with $a=b=0.0001$ (thick dotted line), with a mixture prior (dashed line), with a hyperprior on $\delta$ (dotted line), with adaptive penalties and a mixture prior (solid line) and with adaptive penalties and a hyperprior on $\delta$ (dash-dotted line). Graph(b) : fitted curves with this last model, obtained for some iterations of the MCMC sampler.

tive penalties are required to get a satisfactory fit for such curves. Graph(b) shows fitted curves obtained using the Bayesian P-splines model with adaptive penalties and a hyperprior on $\delta$, for some iterations of the MCMC sampler.

The relative goodness of fit of the competing models can be assessed using the Deviance Information Criterion (DIC) (Spiegelhalter et al., 2002). DIC is defined as a classical estimate of fit plus twice the effective number of parameters:

$$
D I C=D(\boldsymbol{y}, \tilde{\boldsymbol{\beta}})+2 p_{D},
$$

with

$$
D(\boldsymbol{y}, \boldsymbol{\beta})=-2 \log p(\boldsymbol{y} \mid \boldsymbol{\beta}), p_{D}=\boldsymbol{E}_{\boldsymbol{\beta} \mid \boldsymbol{y}}[D(\boldsymbol{y}, \boldsymbol{\beta})]-D(\boldsymbol{y}, \tilde{\boldsymbol{\beta}}),
$$

where $\tilde{\boldsymbol{\beta}}=\boldsymbol{E}(\boldsymbol{\beta} \mid \boldsymbol{y})$ is the posterior mean of the model parameters $\boldsymbol{\beta}$. The effective number of parameters, $p_{D}$, can be estimated by using the following approximation :

$$
\boldsymbol{E}_{\boldsymbol{\beta} \mid \boldsymbol{y}}[D(\boldsymbol{y}, \boldsymbol{\beta})] \approx \frac{1}{M} \sum_{m=1}^{M} D\left(\boldsymbol{y}, \boldsymbol{\beta}^{(m)}\right)
$$




\begin{tabular}{cccccc} 
& Model 1 & Model 2 & Model 3 & Model 4 & Model 5 \\
\hline DIC & 108.47 & 105.41 & 105.86 & 60.76 & 59.99 \\
$p_{D}$ & 8.18 & 6.92 & 6.95 & 7.86 & 8.02
\end{tabular}

Table 3: Application on real data : DIC and $p_{D}$ for a Bayesian P-splines model with -1- $a=b=0.0001 ;-2$ - a mixture prior ; -3- a hyperprior on $\delta ;$-4- adaptive penalties with a mixture prior $;-5$ - adaptive penalties with a hyperprior on $\delta$.

where $M$ is the number of MCMC iterations. For our specification, the deviance is

$$
D(\boldsymbol{y}, \boldsymbol{\theta}, \tau)=-n \log (\tau)+n \log (2 \pi)+\tau(\boldsymbol{y}-B \boldsymbol{\theta})^{\prime}(\boldsymbol{y}-B \boldsymbol{\theta}) .
$$

At each iteration of the MCMC sampler, values for $\tau$ and $\boldsymbol{\theta}$ are generated and $D\left(\boldsymbol{y}, \boldsymbol{\theta}^{(m)}, \tau^{(m)}\right)$ is computed.

$D I C$ is obtained by taking $2\left[\frac{1}{M} \sum_{m=1}^{M} D\left(\boldsymbol{y}, \boldsymbol{\theta}^{(m)}, \tau^{(m)}\right)\right]$ minus the deviance estimated at the posterior mean of $\tau$ and $\boldsymbol{\theta}$.

Table 3 gives the $D I C$ and $p_{D}$ for the different models used in Fig. 15: the best models are the ones combining adaptive penalties with a mixture prior or a hyperprior on $\delta$.

\section{Discussion}

In Bayesian P-splines models, the prior distribution for the roughness penalty parameter is usually taken to be a gamma with hyperparameters $a=1$ and a small value for $b$, or with $a=b$ equal to small value in order to have a large prior variance. We have shown that the choice of these hyperparameters can have a critical influence on the smoothness of the resulting fit in some specific circumstances, i.e. when the sample size or the signal-to-noise ratio is small. This was confirmed by a simulation study involving simple functions like linear, quadratic or sine ones. When the sample size is large, a sensitivity analysis for the choice of the hyperparameters often leads to the conclusion that results 
hardly depend on it. However, we have provided an illustration where the number of observations is large and where the choice of the hyperparameters still has an influence on the fit. In order to spare a likely useless sensitivity analysis and to warrant against the possible consequences of its neglect, it is desirable to make the fitting procedure automatic. The two alternative Bayesian P-splines models proposed in this paper do not require a sensitive choice of hyperparameters in the prior distribution. The simulation study suggests that the mixture prior approach performs slightly better than the hyperprior one, with larger computation times for the first one.

We have also provided an extension enabling adaptive penalties. Combined with one of the two proposed specifications for the reference roughness penalty prior distribution (see Section 3), we end up with a very powerful, easy to set up (cf. Gibbs sampler) and quick Bayesian smoother.

\section{Acknowledgements}

Astrid Jullion thanks Eli Lilly for financial support through a patronage research grant and the UCL for a FSR research grant. Financial support from the IAP research network nr P5/24 of the Belgian State (Federal Office for Scientific, Technical and Cultural Affairs) is also gratefully acknowledged by Philippe Lambert.

Finally, the authors are grateful to the referees for their helpful comments and suggestions.

\section{References}

Baladandayuthapani, V., Mallick, B., and Carroll, R. J. (2005). Spatially adaptive Bayesian penalized regression splines. Journal of Computational and Graphical Statistics, 14, 378-394.

Berry, S. M., Carroll, R. J., and Ruppert, D. (2002). Bayesian smoothing and regression splines for measurement error problems. Journal of the American Statistical Association, 97, 160-169. 
Brezger, A. and Lang, S. (2006). Generalized structured additive regresion based on Bayesian P-splines. Journal of Computational Statistics and Data Analysis, 50, 967-991.

Denison, D., Smith, A. F. M., and Mallick, B. K. (2002). Bayesian methods for nonlinear classification and regression. Wiley, Chichester, West Sussex, England.

Eilers, P. H. C. and Marx, B. D. (1996). Flexible smoothing with B-splines and penalties (with discussion). Statistical Science, 11, 89-121.

Fahrmeir, L., Kneib, T., and Lang, S. (2004). Penalized structured additive regression for space-time data: a Bayesian perspective. Statistica Sinica, 14, $715-745$.

Lambert, P. (2005). Archimedean copula estimation using Bayesian splines smoothing techniques. Discussion Paper 05-27, Institut de Statistique, Université catholique de Louvain, Louvain-la-Neuve, Belgium. http://www.stat.ucl.ac.be/pub/papers/dp/dp05/.

Lambert, P. and Eilers, P. H. (2005). Bayesian proportional hazards model with time varying regression coefficients: a penalized poisson regression approach. Statistics in Medicine, 24. 3977-3989.

Lang, S. and Brezger, A. (2004). Bayesian P-splines. Journal of Computational and Graphical Statistics, 13, 183-212.

Ruppert, D., Wand, M. P., and Carroll, R. J. (2003). Semiparametric regression. Cambridge University Press, Cambridge, UK.

Speckman, P. and Sun, D. (2001). Bayesian nonparametric regression and autoregression prior. Technical report, Department of Statistics, University of Missouri. (http://www.stat.missouri.edu/ speckman/pub.html).

Spiegelhalter, D., Best, N., Bradley, P., and van der Linde A., A. (2002). Bayesian measures of model complexity and fit. Journal of the Royal Statistical Society, Series B, 64, 583-639. 
van Warde, A. (2000). Measuring receptor occupancy with PET. Current Pharmaceutical Design, 6, 1593-1610.

Wahba, G. and Wold, S. (1975). A completly automatic french curve : Fitting spline function by cross validation. Communications in Statistics, 4, 1-18.

Wecker, E. and Ansley, C. (1983). The signal extraction approach to nonlinear and spline smoothing. Journal of the American Statistical Association, 78, $81-89$. 